\title{
Space weather and space anomalies
}

\author{
L. I. Dorman ${ }^{1,2}$, N. Iucci ${ }^{3}$, A. V. Belov ${ }^{2}$, A. E. Levitin ${ }^{2}$, E. A. Eroshenko ${ }^{2}$, N. G. Ptitsyna ${ }^{4}$, G. Villoresi ${ }^{3}$, \\ G. V. Chizhenkov ${ }^{2}$, L. I. Gromova ${ }^{2}$, M. Parisi ${ }^{3}$, M. I. Tyasto ${ }^{4}$, and V. G. Yanke ${ }^{2}$ \\ ${ }^{1}$ Israel Cosmic Ray and Space Weather Center, affiliated to Tel Aviv University, Technion, and Israel Space Agency, Israel \\ ${ }^{2}$ IZMIRAN, Russian Academy of Science, Moscow, Russia \\ ${ }^{3}$ Dipartimento di Fisica E. Amaldi, Roma-Tre University, Rome, Italy \\ ${ }^{2} \mathrm{SPb}$ FIZMIRAN, Russian Academy of Science, St. Petersburg, Russia
}

Received: 25 February 2005 - Revised: 28 May 2005 - Accepted: 24 May 2005 - Published: 22 November 2005

Part of Special Issue "1st European Space Weather Week (ESWW)"

\begin{abstract}
A large database of anomalies, registered by 220 satellites in different orbits over the period 1971-1994 has been compiled. For the first time, data from 49 Russian Kosmos satellites have been included in a statistical analysis. The database also contains a large set of daily and hourly space weather parameters. A series of statistical analyses made it possible to quantify, for different satellite orbits, space weather conditions on the days characterized by anomaly occurrences. In particular, very intense fluxes ( $>1000 \mathrm{pfu}$ at energy $>10 \mathrm{MeV}$ ) of solar protons are linked to anomalies registered by satellites in high-altitude $\left(>15000 \mathrm{~km}\right.$ ), near-polar (inclination $>55^{\circ}$ ) orbits typical for navigation satellites, such as those used in the GPS network, NAVSTAR, etc. (the rate of anomalies increases by a factor $\sim 20$ ), and to a much smaller extent to anomalies in geostationary orbits, (they increase by a factor $\sim 4$ ). Direct and indirect connections between anomaly occurrence and geomagnetic perturbations are also discussed.
\end{abstract}

Keywords. Interplanetary physics (Cosmic rays; Energetic particles; Instruments and techniques)

\section{Introduction}

In this paper we present results obtained in the frame of the INTAS European project on the effect of perturbed space weather conditions on artificial satellites. A body of evidence has been accumulated over the last few decades on the existence of anomalies in spacecraft operation caused by adverse space environment conditions (McPherson and Schober, 1975; Shaw et al., 1976; Lanzerotti, 1979; Allen and Wilkinson, 1993; Stephen, 1993; Feynman and Gabriel, 2000). Anomalies have been associated with intense fluxes

Correspondence to: L. I. Dorman

(lid@physics.technion.ac.il) of energetic particles inside the Earth's magnetosphere (Farthing et al., 1982; Gussenhoven et al., 1985; Fredrickson, 1996; Pease, 1996; Baker et al., 1998), as well as with energetic events connected to geomagnetic storms or auroral substorms (Baker, 1984; Allen et al., 1982, 1989; Shea et al., 1992; Blake et al., 1997; Fennell et al., 2000a).

Analysis of available information has allowed the identification of space weather conditions and mechanisms producing adverse effects on satellite operation (see Feynman and Gabriel, 2000, and references therein). Fluxes of 10 $100 \mathrm{KeV}$ electrons, which can be particularly intense during magnetospheric substorms, can give rise to spacecraft surface charging. Increases in trapped magnetospheric electrons with $\mathrm{E}>100 \mathrm{KeV}$ can cause deep dielectric charging and background counting in sensors. Trapped protons of $0.1-1 \mathrm{MeV}$ produce surface damage to materials of satellites, while increases in $1-10 \mathrm{MeV}$ proton population increase the displacement damage in solar cells. At higher energies intense proton fluxes, mainly of solar origin, increase ionization, displacement damage and sensor background, and for E $>50 \mathrm{MeV}$ single-event effects are generated. Rapid and large changes in the ambient geomagnetic field can cause satellite disorientation, reverse satellite momentum wheel energy transfer, induced currents in conductors, and interfere with on-board, self-oscillating frequency standards. Moreover, the Earth's atmosphere is known to expand at times of increased energy input during the arrival of solar particle storms. Then, low altitude satellites are more likely to collide with atmospheric particles. This decreases their orbital altitude and can lead to a temporary loss of communication, and other more serious effects.

As listed here, there is a rich set of possible interactions between the space environment and spacecraft, which may cause problems in spacecraft performance. Moreover, different adverse space conditions affecting spacecraft operation can be found in different magnetospheric regions (Vampola, 
1994). As a consequence, the interactions in low Earth orbit (LEO) spacecraft are expected to be different from those occurring for high orbits, as in the geostationary satellites (GEO) (Hastings, 1995). Since for GEO the ambient Debye length is much larger than the spacecraft dimension, the plasma behaves as an ensemble of isolated charged particles. The environmental plasma in LEO behaves as a collective medium, since the Debye length is generally smaller than the spacecraft. The plasma in LEO is much more dense and generally much colder than at high altitude orbits. High energy solar particles can easily penetrate to high altitude orbits, but not up to equatorial LEO. In addition, polar LEO spacecraft cross the auroral oval regions above latitudes of $\sim 60^{\circ}$, where high-energy particles can often be encountered.

The adverse influence of space weather conditions on satellite systems may be combated by designing improved satellites and satellite electronics that can withstand the dangerous effects of space weather. On the other hand, comprehensive statistical studies, which are based on large numbers of events, may provide a reliable basis for a quantitative prognosis of hazardous conditions and time scales that would permit operators to take preventive measures to decrease the probability of satellite upsets (Feynman and Gabriel, 2000).

Statistical studies that are conducted on the basis of individual or an assortment of satellites, mostly in geostationary orbits (see, e.g. Farthing et al., 1982; Wilkinson, 1994; Vampola, 1994), have shown that the number of some types of specific anomalies increased in periods of intense geomagnetic activity. Moreover, the portion of the satellite trajectories where faulty operations occurred is located in magnetospheric areas in which the most prominent electromagnetic disturbances and particle fluxes are observed (Lanzerotti at al., 1967; Koons and Gorney, 1991). The distributions of anomaly occurrence time for different GEO spacecraft show that most anomalies are clustered in the midnight to morning sector (in Local Time) (McPherson and Schober, 1975; Fennell, 1982; Fennell et al., 2000a, 2000b). This is associated with the main peculiarity of the structure of magnetospheric fields and currents, and with related increases in electromagnetic disturbances and particle fluxes between midnight and the early morning hours. Local-time dependent anomalies, (mostly observed at GEO), were almost certainly due to lower energy electrons $(10-15 \mathrm{KeV})$ injected into the magnetosphere that caused differential surface charging, resulting in electrostatic discharges as a result of auroral substorms or geomagnetic storms (see Farthing et al., 1982).

\section{The database}

Our database comprises data on anomalous behavior in the operation of the Kosmos series of Russian satellites (1971-1997) and of other spacecraft (19711994) accessible via the Internet (http://hea-www.harvard. edu, http://nssdc.gsfc.nasa.gov/omniweb/ow.html, (OMNI Database); http://ftp.ngdc.noaa.gov/stp/solar_data/, http:// www.ngdc.noaa.gov/stp/goes/anom5jt.txt) and through cata- logues (Wilkinson, 1994; Thomas, 1995). In principle, an inconsistent data set is obtained by the combination of anomalies registered on different spacecraft. Anomalies in spacecraft operation are compiled for various purposes, with different projects catalogue and record anomalies in different formats. Different scoring criteria in anomaly registering can be chosen for different projects. Typically, there are 1-10 anomalies reported per spacecraft per year. However, if one chooses anomaly criteria to include even improper temporary operation of a subsystem, then hundreds of events per year may be found in one satellite (Leung et al., 1986; Koons and Gorney, 1991; Violet and Fredrickson, 1993).

\subsection{Kosmos data}

The Kosmos satellites involved in our database are 49 lowEarth-orbit satellites with a circular orbit at $800 \mathrm{~km}$ and an inclination of $74^{\circ}$. They are in orbits similar in altitude, but with less polar inclination, to the US weather and space environment satellites DMSP and NOAA-POES. Kosmos anomalies were registered and reported in the same manner for all satellites. There were 459 anomalies registered during 1971-1997. For the majority of Kosmos satellites, only the day of anomaly occurrence was registered; thus, our data set is organized on a daily basis.

\subsection{Combined database}

The database consists of three sets of data. The first set reports all anomaly events. For each anomaly we have the following parameters: date and time (universal and local), altitude, latitude and longitude of the satellite (only the date is reported for Kosmos), the type and a short description of the anomaly. The second set is the list of satellites. For each satellite this list contains the working period (starting and ending dates), perigee, apogee, inclination, mean altitude, satellite mass and other special remarks. The third set reports related daily information, such as the number of active satellites, the number of satellites reporting anomalies, the absolute number $(N)$ and the normalized frequency $(n)$ of anomalies. The normalized frequency $n$, in a certain time interval $T$, is calculated as $n=N /(M \bullet T)$, where $N$ is the count of all anomalies registered in the time $T$ by the $M$ operating satellites.

In addition to the anomalies, the database includes the following space weather data:

- Sunspot numbers W and radio-emission flux $(10.7 \mathrm{~cm})$; solar wind velocity and interplanetary magnetic field intensity, geomagnetic activity indices (aa, $A_{p}, A E$ and $D_{s t}$, daily mean, maximum and minimum values);

- Proton daily fluencies (energies $>1,>10,>100 \mathrm{MeV}$ ), measured by GOES satellites during 1987-1994 and hourly fluxes (energies $>10,>60 \mathrm{MeV}$ ), measured by IMP-8 in 1971-1994; 
- Electron daily fluencies (energies $>2 \mathrm{MeV}$ ) and hourly fluxes (energies $>2 \mathrm{MeV}$ ), measured by GOES satellites during 1986-1994;

- Hourly density and maximal daily range of variations of cosmic rays with rigidity $>10 \mathrm{GeV}$, calculated by the global survey method based on neutron monitor network data (Belov et al., 1983); hourly and daily cosmic ray activity indices (Belov et al., 1999a,b).

The combined database comprises about 5700 anomalies in the operation of 220 spacecraft. The 220 satellites have been divided into four groups according to their altitude (high: $>15000 \mathrm{~km}$; low: $<1500 \mathrm{~km}$ ) and their inclination (high: $>55^{\circ}$, low: $<35^{\circ}$ ): 13 high-altitude and highinclination $(\mathrm{HH}), 136$ high-altitude and low-inclination (HL, all GEO), 66 low-altitude and high inclination (LH, 49 are Kosmos), only 5 low-altitude and low inclination (LL). The distribution of the anomalies among the 4 groups is: 1,036 anomalies in group HH, 3.448 in HL, 1.047 in LH, and 152 in LL. Representative satellites in different groups are as follows:

- In the HL group: GOES, SCATHA, ANIK, communication satellites INTELSAT, MARECS-A, TDRSS, European METEOSAT; all satellites in this group operate in geostationary orbits.

- In the HH group: navigation satellites such as those used in the GPS network, NAVSTAR, etc., which typically operate at half-stationary altitude, which places them in a vulnerable orbit (in our database the greatest number of anomalies per satellite occurred in this group).

- In the LH group: Kosmos, SAMPEX, USA meteorological satellites DMSP, NOAA, TIROS.

- In the LL group: mostly manned Shuttles. Since the number of anomalies in this group is not sufficient for a statistically significant analysis, the data of this group will be used only in a few analyses of the combined low altitude group $(\mathrm{LL}+\mathrm{LH})$.

\section{Relationships between space environment and anomalies}

\subsection{General statistics for anomalies in all orbits}

The problem of identifying space environment parameters, which can be related to anomalous behavior in spacecraft operation, will be examined over the years of our combined database (1971-1994). As a first approach the total data set of anomalies registered in all types of spacecraft missions was considered. The great number of events in our database allowed us to compare, by statistical investigation of space weather characteristics, the days with and without satellite anomalies. For this purpose, we divided all of the days into three groups according to the number of satellites registering anomalies: "quiet" days, no anomalies reported; "probably dangerous" days, anomalies reported for one or two satellites; "dangerous" days, anomalies reported for $\geq 3$ satellites. For the last group, we are confident that the anomalies are most likely to be associated with adverse space conditions.

Table 1 demonstrates a general tendency for anomalies to occur in days with enhanced parameters, which characterize disturbed conditions in space environment. This general tendency is seen for the total data set comprising anomalies registered by all types of satellites in all orbits. From this table we see that the geomagnetic indices $A_{p}$ and $D_{s t}$ show a moderate but clear increase (by 30-40\%), going from "quiet" to "probably dangerous" and to "dangerous" days. Moreover, for each group, the daily $A_{p}$ and $D_{s t}$ indexes have a standard deviation comparable to the corresponding average value which is much larger than the observed average changes from "quiet" to "dangerous" days. Therefore, the average trend from "quiet" to "dangerous" days is statistically proven to be real, while, for the individual days, no direct connection can be given between the satellite anomaly occurrence and the increase in geomagnetic parameters. A much more pronounced increase (by a factor from 2.5 to $\sim 50$, from "quiet" to "dangerous" days) is observed for intense proton and electron fluxes, confirming their efficiency in producing satellite anomalies. In these last statistical distributions, the day-to-day fluctuations of the investigated parameters are very large; each distribution has a standard deviation from 2 to 5 times larger than its average value.

All previous results seem to be linked to the nonhomogenous properties of satellites, as confirmed by a separate analysis which showed a lack of correlation between daily anomaly rates in satellites located in different orbits (see the group definition given in Sect. 2.2.). For instance, throughout the period 1975-1994, there were 948 days with $\geq 2$ satellite anomalies at high altitudes and 154 days at low altitudes. Only 11 days from these subsets coincide. Correlation between the daily occurrence of anomalies in any two different groups is very low (correlation coefficients $r<0.002$ ) for any long enough period ( 3 years or more). The only exception occurred in the 1992-1994 years in which $r$ increased up to 0.19 .

We have to consider that different orbits are dominated by different environments: (i) energetic trapped protons from about $\mathrm{L}=1.3$ to $\mathrm{L}=1.8$ (the McIlwain $\mathrm{L}$ parameter, measured in Earth radii, is the characteristic parameter of L-shells; it is the distance from the Earth's surface of the intersection of a geomagnetic dipole field line with the geomagnetic equator); (ii) energetic electrons from about $\mathrm{L}=3$ to $\mathrm{L}=7$; (iii) solar protons, and (iv) hot plasma, mostly at high altitude and in the aurorally zone (low geomagnetic rigidity). However, it should be noted that most of these agents tend to be enhanced during geomagnetically active periods. These periods may last several days. The averaging was done by the epoch method for 388 magnetic storms with maximal $A_{p}>50$. It was found that the average tendency for the total data set of satellite anomalies was to increase in the geomagnetic perturbed periods. However, details of the relationships between 
Table 1. Average characteristics of space weather in days with and without satellite anomalies (1971-1994).

\begin{tabular}{lccc}
\hline Parameter & $\begin{array}{c}\text { "quiet" days } \\
\text { (no anomalies) }\end{array}$ & $\begin{array}{c}\text { "probably dangerous" days } \\
\text { (anomalies in 1-2 satellites) }\end{array}$ & $\begin{array}{c}\text { "dangerous" days } \\
\text { (anomalies in } \geq 3 \text { satellites) }\end{array}$ \\
\hline Total No. of days & 5862 & 2606 & 298 \\
No. of anomalies (per day, per satellite) & 0 & $1.68 \pm 0.04$ & $4.55 \pm 0.18$ \\
No. of satellites with anomalies (per day) & 0 & $1.24 \pm 0.01$ & $3.51 \pm 0.06$ \\
Daily $A_{p}$ & $14.57 \pm 0.18$ & $17.55 \pm 0.36$ & $21.15 \pm 1.32$ \\
Maximal $A_{p}$ & $29.26 \pm 0.40$ & $34.46 \pm 0.73$ & $40.03 \pm 2.53$ \\
Minimal $D_{s t}, \mathrm{nT}$ & $-31.78 \pm 0.38$ & $-36.49 \pm 0.70$ & $-42.68 \pm 2.20$ \\
Daily proton flux $>10 \mathrm{MeV}, \mathrm{pfu}$ & $0.30 \pm 0.09$ & $0.46 \pm 0.12$ & $17 \pm 12$ \\
Maximal proton flux $>10 \mathrm{MeV}, \mathrm{pfu}$ & $8.20 \pm 1.70$ & $18.1 \pm 4.4$ & $91 \pm 30$ \\
Electron fluence $>2 \mathrm{MeV}(\times) 10^{7}$ ), $\mathrm{cm}^{-2}$ & $4.90 \pm 0.29$ & $7.59 \pm 0.60$ & $12.7 \pm 2.7$ \\
Solar wind speed, $\mathrm{km} / \mathrm{s}$ & $441.9 \pm 1.5$ & $466.2 \pm 2.5$ & $500 \pm 9$ \\
IMF intensity, $\mathrm{nT}$ & $6.88 \pm 0.04$ & $6.98 \pm 0.06$ & $6.72 \pm 0.18$ \\
\hline
\end{tabular}

anomalies and space parameters in various orbits may be different according to different physical processes in the Earth's magnetosphere. Hence, we will study these relationships separately for different satellite orbits.

\subsection{Statistical analysis for different satellite orbits}

\subsubsection{Geomagnetic effects}

As mentioned in the Introduction, links between geomagnetic activity and spacecraft anomalies have been reported. Among these links, seasonal effects in specific types of anomalies in geostationary satellites (GOES, MARECS-A, and to some extent in DRA-delta), and increases in anomalies during major geomagnetic storms were observed (Allen and Wilkinson, 1993; Wilkinson, 1994; Wrenn and Sims, 1993; Wrenn et al., 2002). Statistical analyses, to confirm and quantify these links, together with the investigation of their peculiar features in different orbits, will be conducted in this section.

The average annual behavior of anomaly occurrence in different orbits and of geomagnetic activity, characterized by the planetary daily index $A_{p}$ and by the aurorally electrojet index $A E$, was computed for the 1975-1994 period. Yearly curves for the HL group and for the $A_{p}$ index have clear maxima in March and September close to equinoxes. The semiannual variation of geomagnetic activity, consisting of two maxima around equinoctial months, has been known for over 100 years (e.g. Cortie, 1912; Chapman and Bartels, 1940). Historically, several possible mechanisms have been proposed to explain this semiannual variation of geomagnetic activity. The most popular one, the Russell-McPherron effect (Russell and McPherron, 1973), is based on the assumption that geomagnetic activity is at a maximum when the interplanetary magnetic field has the maximal southward component in the solar-magnetospheric coordinate system (that occurs near equinoxes). The two peaks in the yearly behavior of HL (GEO) spacecraft anomalies can be associated with similar increases in geomagnetic activity; part of the anomaly increase can be attributed to increases inside the magnetosphere of energetic solar flare particles in equinox periods. In this context it should be mentioned that similar seasonal peaks of energetic electrons around the equinoxes were observed in GOES $>2 \mathrm{MeV}$ electrons during 19961997 (solar minimum) (Baker et al., 1997; Allen, 2000).

Spacecraft anomalies for the $\mathrm{LH}$ and $\mathrm{HH}$ groups do not show a semiannual variation with anomaly peaks near equinoxes. The average yearly behavior of LH spacecraft (low-altitude, high-inclination orbits) anomalies shows an annual variation. Recent statistical studies by Cliver et al. (2000), Ahn et al. (2000), Lyatsky and Tan (2003) demonstrated strong differences in the seasonal variations of various geomagnetic activity indexes. The bimodal seasonal variation is very strong for the low- and middle-latitude indexes $D_{s t}$ and $A_{p}$, and it is absent in the $A E$ index, which is a measure of geomagnetic activity in the auroral zone and is related to substorms. The $A E$ index for the considered years shows an annual variation which can be attributed to the annual variation in ionospheric conductivity with a peak in summer months. It is evident that geomagnetic activity in the auroral zone contributes greatly to the seasonal behavior of anomalies of spacecraft traveling in highly inclined orbits.

Further, a statistical analysis has been applied to verify links between satellite anomalies $n$ and intense geomagnetic storms with SSC. A total of 441 SSC geomagnetic storms (maximum $A_{p} \geq 40$ ), occurring during the analyzed period, have been considered. The average $n$ values, in the days preceding and following the sudden commencement of geomagnetic storms, for both high (HL and $\mathrm{HH}$ ) and lowaltitude (LH and LL) satellites, has a tendency to rise to about 
0.016 anomalies per day, per satellite, which represents an increase by a factor 1.5-2.0 with respect to the pre-SSC level. However, the time profile of this rise is different for different orbits. At high altitudes, the rise starts one day after the SSC occurrence and extends mainly during the main phase of geomagnetic storms. At low altitudes, the rise starts on the third day after the SSC and reaches a maximum on the 5th day; in this case the most unfavorable period for satellite electronics occurs after the main phase of geomagnetic storms. These results are indicative of the effect of various sources on the observed anomalies for different satellite orbits.

\subsubsection{Proton flux effect}

To study linkages between proton events and satellite anomalies, the superposed epoch analysis was applied by choosing the proton event onset as zero-day. At first, we considered all solar proton events at energy $\geq 10 \mathrm{MeV}$, under the requirement of a maximal proton flux $\mathrm{F}>10 \mathrm{pfu}$, which is slightly higher than the average maximum flux measured during quiet days (see Table I). Similar behavior in the normalized frequency of anomalies $n$ is found for both low (LL and LH) and high-altitude (HH and HL) satellites. The frequency increases slowly, reaching a maximum value 4-5 days after the zero-day. This occurs presumably when the proton event has ended and the interplanetary perturbation (shock and flareejecta ion cloud), produced by the flare, associated with the enhanced proton flux, has reached the Earth.

A completely different situation is found to occur when only the greatest eight proton events (maximal flux $\mathrm{F}>1000 \mathrm{pfu}$ ) are taken into consideration. For high-altitude satellites, $n$ rises by a factor $\sim 10$ in the zero and first days, just at the beginning of proton events. On the contrary, no significant increase is observed in low-altitude orbits. The dependence of $n$, for different orbits, on the maximum flux involved in the proton events, shows no increases rise above the statistical fluctuations in the case of low-altitude satellites; a moderate increase for very intense fluxes $F>1000$ pfu is observed for HL (GEO) orbits; a very large effect emerges for $\mathrm{HH}$ satellites. In this last group the average $n$ increases at $\mathrm{F}>100 \mathrm{pfu}$, reaching $\sim 0.19$ for the 8 largest solar proton events $(\mathrm{F}>1000 \mathrm{pfu}$, i.e. a factor $\sim 20$ higher than the frequency at $\mathrm{F}<10 \mathrm{pfu}$ ). For this $\mathrm{HH}$ spacecraft group a $60 \%$ probability to realize an anomaly is reached for $\mathrm{F}>300 \mathrm{pfu}$ at proton energy $>60 \mathrm{MeV}$, while a $30 \%$ probability is reached for $\mathrm{F}>1000$ pfu at $\mathrm{E}>10 \mathrm{MeV}$.

\subsubsection{Electron flux effect}

The epoch method was used to study relationships between satellite anomalies and intense fluxes of relativistic electrons $(\mathrm{E}>2 \mathrm{MeV})$, as observed by GOES satellites at geostationary orbit. Since it is not easy to define the beginning of electron events, the day of each satellite anomaly was selected as zero-day, and the average behavior of the electron flux at GOES about the zero-day has been investigated. For all geostationary satellites a clear increase by a factor $\sim 2$ is found. We notice that the average general level of electron fluency is found for the whole period from -12 to +12 days, much higher than the average level for "quiet" days (see the Table 1). This is an indication of the occurrence of GEO anomalies in long-lasting series. A statistically significant effect has also been found for Kosmos, but no effect is observed for HH orbits. For the GEO group a large portion of all anomalies is observed inside these periods of increased flux of relativistic electrons. On average, this flux is found to increase a week before and a week after the anomaly days. These results confirm, with a high statistical level, previous reports showing that long-lasting, high-energy electron fluxes are the cause of a large part of anomalies at geosynchronous orbits due to electrostatic discharges (Vampola, 1987; Koons and Gorney, 1991; Fredrickson, 1996; Baker et al., 1998, Koons et al., 1999; Fennel et al., 2000a). Energetic electrons $(>300 \mathrm{KeV})$ are highly variable in the inner and outer magnetosphere, and their enhancements have been associated with long-lived high-speed solar wind streams, as well as with recurrent geomagnetic storms (Paulikas and Blake, 1979; Baker et al., 1986, 1997). These energetic electron enhancements are often associated with the late part of a geomagnetic disturbance. Recently, it has been shown that such an enhancement in the energetic electron fluxes also requires a southward-directed interplanetary magnetic field (Blake et al., 1997). The interplanetary magnetic field has the maximal southward component in the solar-magnetospheric coordinate system near the equinoxes. A similar seasonal dependence in the occurrence rate of anomalies for HL satellite groups has been reported in Sect. 3.2.1. In this regard, the result of a superposed epoch analysis of $A_{p}$ and $A E$ indexes, taking zero epoch anomaly days at GEO satellites, shows that, on average, these anomaly days are located towards the end of geomagnetic perturbed periods. For days when anomalies in GEO satellites occurred, there is an $A E$ increase (a substorm index). During substorms, hot plasma can be injected into the nightside of high-altitude equatorial regions. The freshly injected electrons (from a few hundreds of $\mathrm{eV}$ to several $\mathrm{KeV}$ ) can lead to an increase in satellite surface charging and anomalies. Farthing et al. (1982) found a similar time delay between the injection of plasma (substorm onset) in the midnight sector and the arrival of energetic electrons at the satellites, which produced phantom commands in GOES-4 and 5. They found a regular linear distribution of charging events plotted against the delay after sub-storm onset. The slope of the best-fit line corresponded to the drift velocity of $10-15 \mathrm{KeV}$ electrons injected on the midnight meridian and drifting toward dawn until they encountered the GOES satellite. A portion of the anomalies at geostationary orbits used in our analysis was certainly due to this surface charging. A clear seasonal variation in $\mathrm{HL}$ anomalies supports this mechanism.

A separate analysis of Kosmos data in relation to geomagnetic activity is given in Sect. 3.3. 


\subsubsection{Examples of disturbed space weather effects}

In this section peculiarities in anomaly occurrences are discussed for individual disturbed space weather periods and compared with the statistical results.

The intense solar proton events of October 1989:

We analyzed the space environment and satellite anomaly occurrences in the time interval 15-31 October 1989, in which a series of prominent solar activity events occurred. Three intense proton enhancements were observed at ground level (GLE events) on 19, 22 and 24 October and are the main feature of the period; moreover, large Forbush decreases and strong geomagnetic storms took place. Both $\mathrm{HH}$ and HL (GEO) satellites show a remarkable increase in anomaly rates, which are mostly concentrated in the periods of the highest levels of proton enhancements. According to our database, during this disturbed period, 73 anomalies were reported by different satellites (due to overlapping, not all of them are visible in the plot). Only one anomaly was registered in low orbit at high inclination (LH) by a Kosmos satellite and 19 anomalies were registered in GEO orbits (HL); the majority of the anomalies (53) were reported by satellites in high altitude orbits with high inclinations $(\mathrm{HH})$, i.e. in the satellite group which is maximally exposed to solar cosmic rays. It has to be noted that for these events $n$ for $\mathrm{HH}$ is 15 times larger than for GEO satellites (the number of operating GEO satellites is 5.5 times larger than the number of $\mathrm{HH}$ satellites during this period). For both HL (GEO) and HH satellites, during the time interval 15-31 October, 1989, about $95 \%$ of the anomalies are concentrated in periods of very intense proton fluxes $(F>1000 \mathrm{pfu}$ at $>10 \mathrm{MeV}$ ), which cover $\sim 30 \%$ of the time with $F>5$ pfu. These values are in agreement with the statistical results of the previous section.

The magnetospheric high-energy electron events of AprilMay 1991 and March 1994:

These periods are characterized by an increasing of satellite anomaly rates associated with intense fluxes of energetic electrons inside the magnetosphere. These electrons are associated with geomagnetic sub-storms and are observed by GOES geostationary satellites in the night-morning portion of their orbits. Since these particles are trapped by the geomagnetic field, they can also reach high latitudes along shells of a constant L parameter. Therefore, these particles affect not only LH but also HL satellites. Geomagnetic activity is represented by $K_{p}$ and $A E$ indices. For April-May 1991 and March 1994 events, $K_{p}$ and $D_{s t}$ indices indicate moderate geomagnetic activity. The $A E$ index, which is a measure of substorm activity, is particularly high during these time intervals; it exceeds the $500 \mathrm{nT}$ level for a large part of the time (ninety percent of the time the hourly $A E$ values are well below the $500 \mathrm{nT}$ (Allen and Kamei, 1979). The selected examples show different behaviors in the anomaly occurrences. The March 1994 event is a long lasting energetic electron event. The electron flux $F$ at
GOES starts on 7-8 March; it reaches values over $300 \mathrm{pfu}$ with peaks over $1000 \mathrm{pfu}$ during the period 9-20 March, and it extends, with lower flux levels, until 28 March. No anomalies were observed by $\mathrm{HH}$ satellites, while increases in anomaly rates occur for HL satellites. The rate of HL anomalies seems to follow the electron flux behavior. The anomaly rate for the LH satellites is much smaller (eight anomalies, one of them reported by Kosmos) and seems to be concentrated in the last part of the event.

The second example of April-May 1991 is quite different. A Forbush decrease is accompanied by a moderate geomagnetic storm started on 25 April; also at this time a moderate increase in proton flux is in progress which ends on day 28. During the recovery phase of this Forbush event, a geomagnetic storm began; this storm lasted for several days. GOES satellites at geostationary orbit observed a series of elevated fluxes of energetic electrons. This periodic electron flux is observed until 8 May, i.e. after the end of the geomagnetic perturbation. The average flux of this event is a factor of 2.5 lower than for the 1994 event. For GEO satellites a lower anomaly rate is observed, as is expected. On the contrary, a very high anomaly rate is observed for LH satellites. This difference may be caused by a peculiar distribution of electrons inside the magnetosphere. In addition, we remark that a single LH satellite (STS-39) is responsible of a large portion of anomalies (six anomalies were reported by Kosmos).

During these two periods of perturbed space weather conditions, characterized by the persistent presence of energetic electrons at geostationary altitudes in the near-equatorial magnetosphere, anomalies were absent at high-altitude with high-inclination satellites, which were found to be the main victims of solar proton precipitation. This is in agreement with the statistical results (see Sect. 3.2.3.), showing that anomalies in HH spacecraft are not linked to electron fluxes at geostationary altitudes.

\subsection{Specific analysis of cosmos satellite anomalies}

The influence of solar and geomagnetic activity on anomalies of Kosmos (polar and low-latitude) satellites was analyzed for the years 1971-1997. The 49 Kosmos satellites that were studied here are a single type. They have the same orbit, and the monitoring and classification of their anomalies has been done in a similar manner. Therefore, Kosmos anomaly data can be considered as a homogeneous group, suitable for accurate studies on their links with solar and geomagnetic activity. Moreover, they have never been used for this purpose until now.

The relative sunspot Wolf number (W) and the $2800 \mathrm{MHz}$ $(10.7 \mathrm{~cm})$ solar flux $(\mathrm{F} 10.7)$ are chosen as solar activity parameters. Selected parameters of geomagnetic activity are the daily indices: $A_{p}$, for the level of planetary activity; $D_{s t}$, for low-latitude disturbances and magnetospheric ring currents; and $A E$, for aurorally disturbances. 


\subsubsection{Cosmos anomalies and the solar activity cycle}

Kosmos anomaly data cover two and a half solar cycles: the declining phase of cycle 20, and two full solar cycles, 21 and 22. This allows us to study the rate of Kosmos anomalies as a function of the phase of the solar activity cycle, which is the dominant driver for many space weather processes. The time behavior of the average yearly normalized rate $n$ of daily Kosmos anomalies is compared with the corresponding behavior of the F10.7 flux. Two main features emerge clearly: a general tendency of $n$ to follow solar activity; and a sharp reduction of anomalies across the 1990-1996 years. This large decrease might be reasonably attributed to the measures taken in those years to diminish environmentallyinduced anomalies in Kosmos satellites. The average normalized anomaly rate $n$ is found to increase with increasing solar activity. An overall increase by a factor $\sim 3$ is observed from low to high solar activity. Therefore, a longterm effect of solar activity on anomaly occurrence on Kosmos satellites clearly exists, but the correlation between $n$ and solar activity (W or F10.7) is rather low (0.7). However, a strong relationship exists between Kosmos anomalies and geomagnetic activity (see the next subsection), which can be perturbed also in years of low solar activity, as in the year 1976 in which the anomaly rate $n$ is particularly high. During the years 1992-1994 of decreasing solar activity, but increasing daily fluxes of high-energy electrons $(>2 \mathrm{MeV})$, a corresponding increase in electrostatic discharges and GEO "DRA-delta" satellite anomalies due to internal charging has been observed (Wrenn, 1995; Wrenn et al., 2002). Relativistic electrons are related to recurrent high-speed streams which reflect the persistence of coronal holes on the Sun. They preferentially occur not at solar maximum, but during the declining phase of solar activity, in which stable and long-lived high-speed solar wind streams are observed. This solar cycle pattern is in agreement with measurements of high-energy electrons $(>2 \mathrm{MeV}$ ) taken during solar cycle 21 (Baker et al., 1993). On the contrary, fluxes of lower-energy $\mathrm{KeV}$ electrons, causing surface charging and related electrostatic discharges, can be more intense during maximum solar activity, in which the occurrence of geomagnetic storms and substorms is most frequent. Wrenn and Smith (1996) utilized MARECS-A anomaly data to illustrate this contrast. The substantial decrease in anomalies in a number of Kosmos satellites after measures were taken to prevent surface charging (see Sect. 2.1) can be considered in this context.

\subsubsection{Kosmos anomalies and geomagnetic activity}

We have already found in the case of geomagnetic storms with SSC, that the average normalized rate of daily anomalies $n$ reaches the maximum value 4-5 days after the SSC; this result indicates the tendency of these anomalies to be delayed by some days with respect to the days of maximum perturbation intensity. The delays of the anomaly days with respect to the maximum perturbation day are also investigated, together with the connection between the maximum daily intensity of the perturbation and the daily anomaly rates $n$. The analysis is done for the three geomagnetic daily indices: $A_{p}, D_{s t}$ and $A E$. As a first step, the distribution of geomagnetic indices about the anomaly days is analyzed. Much higher geomagnetic indices are observed in the anomaly day or in the preceding days, and much lower values in the following days. We found that the majority of the anomalies (55\%) occurs within 0-2 days. However, a part of the anomaly days (27\%) is delayed by $3-5$ days with respect to the day of maximum perturbation. Only $7 \%$ are delayed by 6-8 days. Anomalies delayed by more than 8 days are rare $(11 \%)$ and they could be due to accidental coincidence.

The delayed anomalies are most likely to be triggered by the elevated flux of relativistic electrons through a process of deep dielectric charging, as the persistent intense fluxes of $\geq 2 \mathrm{MeV}$ electrons observed by GOES and associated with severe auroral substorms. The decay time of the dielectric charging depends on the capacitance of the material and may last several hours or days. This delay could also be due to the return of higher levels of high-energy electrons that were depleted during the geomagnetic storm (Wrenn, 1995). Each anomaly day has been associated to the maximum geomagnetic daily index observed during the 10 days preceding the anomaly day.

For all three geomagnetic indices a stable increase was found in the normalized anomaly rate with increasing geomagnetic activity, reaching, in the case of intense perturbations $\left(A_{p}>45, D_{s t}<-120 \mathrm{nT}, A E>700 \mathrm{nT}\right), n>0.05$ anomalies per day per satellite. This value should be compared with the $n \sim 0.003$ average value for geomagnetic quiet periods. The maximum effect is observed in the case of the $A E$ index: the average anomaly level increases from $n=0.003$ anomalies per day per satellite at $A E \leq 200 \mathrm{nT}$, up to $n=0.180$ for $A E>800 \mathrm{nT}$, which represents an increase rate by a factor of 60 . A similar increase of surface-chargingrelated anomalies on high-altitude, high-inclination satellites with heightened geomagnetic activity has been reported by Spence et al. (1993).

It was found that for Kosmos satellites the anomaly rates are better related to those magnetospheric processes whose intensity is described by the $A E$ index, i.e. by substorms and aurorally activity, or, in other terms, by the in-situ presence of an intense population of trapped lower energy $(<300 \mathrm{KeV})$ electrons, which appears to be the direct product of magnetospheric substorm activity. Under appropriate conditions hot auroral electrons ( $300 \mathrm{KeV}>\mathrm{E}>100 \mathrm{eV}$ ) associated with substorm plasma injections observed at geosynchronous orbits can charge low-altitude, polar-orbiting satellites (see Fennel et al., 2000a). This has been established by DMSP satellite charging observations (Gussenhoven et al., 1985; Anderson, 2001) and by the occurrence of anomalies associated with such charging (Anderson and Koons, 1996). Our results on the increase of Kosmos anomalies with heightened geomagnetic activity and its solar cycle dependence fit the pattern of surface charging by $\mathrm{KeV}$ electrons. 


\section{Summary and discussion}

Our research, based on a large amount of data, has provided verification and quantification for linkages between a large fraction of spacecraft anomalies and space weather perturbations and has revealed different peculiarities of these linkages for different orbits.

A database, comprising about 5700 anomalies registered by 220 satellites and covering more than two solar cycles, has been created. Forty-nine Russian Kosmos satellites have been included, for the first time, in this kind of analysis. The use of Kosmos data made it possible to analyze a great number of anomalies in the low-altitude and high-inclination (LH) group of satellites, traveling along orbits crossing the aurorally regions with $L$ values similar to those of GEO satellites, but with a higher rigidity threshold for cosmic rays and energetic solar protons. This database contains, together with satellite anomaly data, basic information on space weather around and inside the Earth's magnetosphere, and on solar activity. Since only a few satellite projects provide onboard measurements of space weather conditions, for most anomalies space weather measurements taken in different environments have been utilized. The large gaps in the distribution of satellites orbits, in altitude (from 1500 to $15000 \mathrm{~km}$ ), as well as in inclination (from $35^{\circ}$ to $55^{\circ}$ ), allowed us to do a simple separation of all satellites into four groups, characterized by very different average altitude and inclination (HL: high-altitude with low-inclination, operating at geostationary orbit; HH: high-altitude with high-inclination, mostly operating at half-stationary altitude; LH: low-altitude with highinclination; LL: low-altitude with low-inclination, mostly manned missions).

Our statistical analysis has shown that there is a tendency for anomalies to occur on days with enhanced activity parameters which characterize disturbed conditions in the space environment. This general tendency is seen for the total data set, comprising all anomalies registered by all types of satellites traveling on all orbits. Among 8766 days studied, there were 2606 days in which 1-2 satellites reported anomalies ("probably dangerous" days), and 298 days during which anomalies were registered on more than 3 satellites ("dangerous" days). During "dangerous" days about $25 \%$ of all anomalies were registered. Geomagnetic indexes $A_{p}$ and $D_{s t}$ show a moderate but clear increase (by 30-40\%) from "quiet" to "dangerous" days. A much more pronounced increase (by a factor from 2.5 to about 50 from "quiet" to "dangerous" days) is observed for near-Earth proton $(\mathrm{E}>10 \mathrm{MeV}$, observed by IMP-8) and electron fluxes ( $\mathrm{E}>2 \mathrm{MeV}$, observed by GOES operating inside the magnetosphere at geostationary orbit). It has to be noted that all these general results are mainly determined by the HL and LH groups which contain altogether about $90 \%$ of all satellites considered in this analysis.

For the HH group the average level of electron flux is found to be at the normal background level, while the proton flux is about 90 times larger. For the LH group the proton flux is at a normal level, while the electron flux is 8 times bigger.
For the HL group, the average fluxes of both energetic electrons and solar protons are high, being respectively 10 and 20 times bigger than the corresponding normal background levels. As a rule the dangerous level of $1000 \mathrm{pfu}$ is reached at least one hour after the beginning of the proton event and $1.5 \mathrm{~h}$ after the beginning of the GLE, which could be used as a predictor of these enhanced proton events (Dorman et al., 2004). Usually, after the increasing phase the proton flux remains enhanced ( $\geq 500 \mathrm{pfu}$ ) for about two days. Intense fluxes of magnetospheric electrons $(\mathrm{E}>2 \mathrm{MeV})$ are observed on days when anomalies were registered in spacecraft travelling on geostationary orbits (HL) and LEO polar orbits (LH), but not on high-altitude, highly inclined orbits (HH).

During geomagnetic storms the anomaly rate increases by a factor $\sim 2$ for all satellites, but particularly for those in the HL and LH orbits.

Acknowledgements. This work depends upon data that have been acquired from a number of sources and we thank all people who made the data available. We would like to thank J. Allen who generously shared with us his knowledge and ideas and G. L. Wrenn for helpful information. This work was supported by INTAS grant 00-0810 and in part by INTAS 2000-752 and RFFR 01-02-17580 grants, and is in the frame of COST 724. Our thanks to both Reviewers for useful comments.

Topical Editor T. Pulkkinen thanks two referees for their help in evaluating this paper.

\section{References}

Ahn, B.-H., Kroehl, H. W., Kamide, Y., and Kihn, E. A.: Seasonal and solar cycle variations of the auroral electrojet index, J. Atmos. Sol. Terr. Phys., 62, 1301-1308, 2000.

Allen, J. H.: Some commonly used magnetic activity indices: their derivation, meaning and use, Proceedings of a Workshop on Satellite Drag, 18-19 March, 1982, NOAA Environmental Research Laboratories, Boulder, CO, 114-134, 1982.

Allen, J. H.: Satellite Anomalies, Recent events and possible causes, SCOSTEP CDROM of COSPAR Colloquium on Space Weather, Taipei, September 2000;

Allen, J., Frank, L., Sauer, H., and Reiff, P.: Effects of the March 1989 solar activity, EOS Trans. AGU 70(46), 1479-1488, 1989.

Allen, J. H. and Wilkinson, D. C.: Solar-terrestrial activity affecting systems in space and on Earth, in: Proceedings of the Workshop Solar-Terrestrial Predictions-IV (Ottawa, Canada, 18-22 May 1992), edited by: Hruska, J., Shea, M. A., Smart, D. F., and Heckman, G., NOAA Environmental Research Laboratories, Boulder, CO, 1, 75-107, 1993.

Allen, J. H. and Kamei, T.: Comparison of geomagnetic activity indices: 1966-1974, in: Proceedings of the Mayaud Simposium, XVIIth General Assembly IUGG, Canberra, 1979.

Anderson, P. C.: A survey of spacecraft charging events on the DMSP spacecraft in LEO, in: Proceedings of the 6th Spacecraft Charging Technology Conference, AFRL-VS-TR20001578, 2001.

Anderson, P. C. and Koons, H. C.: Spacecraft charging anomaly on a low-altitude satellite in an aurora, J. Spacecraft and Rockets, 33, 734-741, 1996. 
Baker, D. N.: Effects of the solar-terrestrial environment on satellite operations, in: Proceedings of the Solar-Terrestrial Predictions Workshop, Meudon, France, LAUR 84-3325, 1984.

Baker, D. N., Blake, J. B., Klebesadel, R. W., and Higbie, P. R.: Highly relativistic electrons in the Earth's outer atmosphere: 1. Life times and temporal history 1979-1984, J. Geophys. Res., 91, 4265-4276, 1986.

Baker, D. N., Goldberg, R. A., Herraro, F. A., Blake, J. B., and Callis, L. B.: Satellite and rocket studies of relativistic electrons and their influence on the middle atmosphere, J. Atmosph. and Terr. Phys., 55, 1619-1628, 1993.

Baker, D. N., Li, X., Turner, N., Allen, J. H., Bargatze, L. F., Blake, J. B., Sheldon, R. B.,. Spence, H. E, Belian, R. D., Reeves, G. D., Kanekal, S. G., Klecker, B., Lepping, R. P., Ogilvie, K., Mewaldt, R. A., Onsager, T., Singer, H. J., and Rostoker, G.: Recurrent geomagnetic storms and relativistic electron enhancements in the outer magnetosphere: ISTP coordinated measurements, J. Geophys. Res., 102, 14 141-14 152, 1997.

Baker, D. N., Allen, J. H., Kanekal, S. G., and Reeves, G. D.: Disturbed space environment may have been related to pager satellite failure, EOS Trans. AGU, 79, 477 and 482-483, 1998.

Belov, A. V., Dorman, L. I., and Yanke, V. G.: The simplest version of the global-spectrographical method, in: Proceedings of Int. Cosmic Ray Conf. 18th, 10, 144-147, 1983.

Belov, A. V., Eroshenko, E. A., and Yanke, V. G.: Indices of the cosmic ray activity as reflection of situation in interplanetary medium, ESA Space Weather Workshop papers, ESTE, 325328, 1999a.

Belov, A. V., Eroshenko, E. A., Yanke, V. G., Antonova, V. I., and Kryakunova, O. N.: Global and local indices of cosmic ray activity, in: Proceedings of Int. Cosmic Ray Conf. 26, 6, 472-475, 1999b.

Blake, J. B., Baker, D. N., Turner, N., Ogilvie, K. W., and Lepping, R. P.: Correlation of changes in the outer-zone relativistic electron population with upstream solar wind and magnetic field measurements, Geophys. Res. Lett., 24, 927-930, 1997.

Chapman, S. and Bartels, J.: Geomagnetism, 1, Oxford Univ. Press, New York, 1940.

Cliver, E. W., Kamide, Y., and Ling, A. G.: Mountains versus valleys: semiannual variation of geomagnetic activity, J. Geophys. Res., 105, 2413-2424, 2000.

Cortie, A. L.: Sunspots and terrestrial magnetic phenomena, 18981911, Mon. Not. R. Astron. Soc., 73, 52-60, 1912.

Dorman L. I., Pustil'nik, L. A., Sternlieb, A., Zukerman, I. G., Belov, A. V., Eroshenko, E. A., Yanke, V. G., Mavromichalaki, H., Sarlanis, C., Souvatzoglou, G., Tatsis, S., Iucci, N., Villoresi, G., Fedorov, Y., Shakhov, B. A., and Murat, M.: Monitoring and Forecasting of Great Solar Proton Events Using the Neutron Monitor Network in Real Time, IEEE Transactions on Plasma Physics, 0093-3813, 1-11, 2004.

Farthing, W. H., Brown, J. P., and Bryant, W. C.: Differential spacecraft charging on the geostationary operational satellites, NASA Technical Memorandum 83908 (43 pages), 1982.

Fennell, J. F.: Description of P78-2 (SCATHA) satellite and experiments, in: The IMS Source Book, edited by: Russell, C. T. and Southwood, D. J., AGU, Washington, D.C., 1982.

Fennell, J. F., Roeder, J. L., and Koons, H. C.: Substorm and magnetic storms from the satellite charging perspective, COSPAR Colloquium, Taipei, 2000a.

Fennell, J. F., Koons, H. C., Chen, M., and Blake, J. B.: Internal charging: a preliminary environmental specification for satellites, IEEE Trans. on Plasma Science, 28, 2029-2036, 2000 b.
Feynman, J. and Gabriel, S. B.: On space weather consequences and predictions, J. Geophys. Res., 105(A5), 10 543-10 564, 2000.

Fredrickson, A. R.: Upset related to spacecraft charging, IEEE Trans. Nucl. Sci., 43, 426-441, 1996.

Gussenhoven, M. S., Hardy, D. A., Rich, F., Burke, W. J., and Yeh, H.-C.: High level spacecraft charging in the low-altitude polar auroral environment, J. Geophys. Res., 90(A11), 11 009-11 024, 1985.

Hastings, D. E.: A review of plasma interactions with spacecraft in low Earth orbit, J. Geophys. Res., 100(A8), 14457-14 483, 1995.

Koons, H. C. and Gorney, D. G.: Relationship between electrostatic discharges on Spacecraft P78-2 and the electron environment, J. Spacecraft and Rockets, 28, 683-688, 1991.

Koons, H. C., Mazur, J. E., Selesnick, R. S., Blake, J. B., Fennell, J. F., Roeder, J. L., and Anderson, P. C.: The impact of the space environment on space systems, Space and Environment Technology Center, Technology Operations, Aerospace Corporation, Report No. TR-99(1670)-1, 1999.

Lanzerotti, L. J.: Geomagnetic influences on man-made systems, J. Atm. Terr. Phys., 41, 787-796, 1979.

Lanzerotti, L. J., Roberts, C. S., and Brown, W. L.: Temporal variation in the electron flux at synchronous altitudes, J. Geophy. Res., 72(23), 3893-3902, 1967.

Leung, P., Wittlesey, A. C., Garret, H. B., and Robinson, P. A.: Environment-induced electrostatic discharges as the cause of Voyager 1 power-on resets. J. Spacecraft and Rockets, 23, 323 330, 1986.

Lyatsky, W. and Tan, A.: Semiannual variation of geomagnetic activity, J. Geophys. Res., 108(A5), 1204-1214, 2003.

McPherson, D. A. and Schober, W. R.: Spacecraft charging at high altitudes: the SCATHA program, in: Spacecraft Charging by Magnetospheric Plasmas, edited by: Rosen, J., Progress in Astronautics and Aeronautics, MIT Press, Cambridge, MA, 47, 15-30, 1975.

Paulikas, J. A. and Blake, J. B.: Effects of the solar wind on magnetospheric dynamics: energetic electrons at the synchronous orbit, in: Quantitative Modeling of Magnetospheric Processes, Geophysics Monograph., edited by: Olsen, W. P., 21, 180, Am. Geophys. Union, Washington, D.C., 1979.

Pease R. L.: Total dose issue for microelectronic in space systems, IEEE Trans. Nucl. Sci., 43, 442-452, 1996.

Russell, C. T. and McPherron, R. L.: Semi-annual variation of geomagnetic activity, J. Geophys. Res., 78, 92-108, 1973.

Shaw, R. R., Nanevicz, J. E., and Adamo, R. C.: Observations of electrostatic discharges caused by differential satellite charging, in: Spacecraft Charging by Magnetospheric Plasmas, edited by: Rosen, J., Progress in Astronautics and Aeronautics, AIAA, New York, 47, 61-76, 1976.

Shea, M. A., Smart, D. F., Allen, J. H., and Wilkinson, D. C.: Spacecraft problems in association with episode of intense solar activity and related terrestrial phenomena during March 1991, IEEE Trans. Nuc. Sci., 39(6), 1754-1760, 1992.

Spence, H. E.,. Blake, J. B, and Fennell, J. F.: Surface charging analysis of high-inclination, high-altitude spacecraft: identification and physics of the plasma source region, IEEE Trans. Nucl. Sci., 40, 1521-1527, 1993.

Stephen, J. H.: Hazard to electronic in space, NATO ASI ser. E., 245, 407, 1993

Thomas, W. B.: Orbital anomalies in Goddard spacecraft for calendar year 1994, NASA technical paper 9636, 1995. 
Vampola, A. L.: Thick dielectric charging on high-altitude spacecraft, J. of Electrostatics, 20(1), 21-30, 1987.

Vampola, A. L.: Analysis of environmentally induced spacecraft anomalies. J. Spacecraft and Rockets, 31, 154-159, 1994.

Violet, M. D. and Frederikson, A. R.: Spacecraft anomalies on the CRRES satellite correlated with the environment and insulator samples. IEEE Trans. Nucl. Sci., 40, 1512-1520, 1993.

Wilkinson, D. C.: NOAA spacecraft anomaly data base and examples of solar activity affecting spacecraft, J. Spacecraft and Rockets, 31(2), 160-165, 1994.

Wrenn, G. L.: Conclusive evidence for internal dielectric charging anomalies on geosynchronous communication spacecraft, J. Spacecraft and Rockets 32, 514-520, 1995.
Wrenn, G. L. and Sims, A. J.: Surface charging of spacecraft in geosynchronous orbit, in: The Behavior of Systems in the Space Environment, edited by: DeWitt, R. N., Duston, D. R., and Hyder, A. K., Kluwer, Dordrecht, The Netherlands, 491-511, 1993.

Wrenn, G. L. and Smith, R. J. K.: Probability factors governing ESD effects in geosynchronous orbit. IEEE TNS 43, 2783-2789, 1996.

Wrenn, G. L, Rodgers, D. J., and Ryden, K. A.: A solar cycle of spacecraft anomalies due to internal charging, Ann. Geophys., 20, 953-956, 2002,

SRef-ID: 1432-0576/ag/2002-20-953. 